http://dx.doi.org/10.35381/r.k.v5i1.778

\title{
Migración y rendimiento académico en estudiantes de bachillerato
}

\section{Migration and academic performance in high school students}

\author{
Julio Xavier Masapanta-Serpa \\ julio.masapanta@psg.ucacue.edu.ec \\ Universidad Católica de Cuenca, Azogues \\ Ecuador. \\ https://orcid.org/0000-0002-7155-7602 \\ Darwin Gabriel García-Herrera \\ dggarciah@ucacue.edu.ec \\ Universidad Católica de Cuenca, Azogues \\ Ecuador \\ https://orcid.org/0000-0001-6813-8100 \\ Luis Bolívar Cabrera-Berrezueta \\ bolivarcabrera@ucacue.edu.ec \\ Universidad Católica de Cuenca, Cuenca \\ Ecuador \\ https://orcid.org/0000-0002-6853-635X \\ Juan Carlos Erazo-Álvarez \\ jcerazo@ucacue.edu.ec \\ Universidad Católica de Cuenca, Cuenca \\ Ecuador \\ https://orcid.org/0000-0001-6480-2270
}

Recibido: 22 de abril de 2020

Revisado: 10 de mayo de 2020

Aprobado: 29 de mayo de 2020

Publicado: 27 de junio de 2020 


\title{
RESUMEN
}

La investigación tuvo por objetivo determinar la influencia que tiene la migración en el rendimiento académico de los estudiantes en cuanto a la evaluación Ser Bachiller. Metodológicamente es tipo descriptiva correlacional no experimental, a través de los datos de 1234 estudiantes, perteneciente a los cantones de Cañar, El Tambo y Suscal del Ecuador. Al aceptarse la hipótesis afirmativa, se comprueba que la motivación a migrar influye positivamente en el rendimiento académico de los estudiantes. La migración cuando es planificada, puede influir positivamente en el rendimiento académico de los estudiantes de bachillerato, por cuanto se perciben con posibilidades de superarse, aunado a generar oportunidades de ingresar a estudios universitarios en el país receptor.

Descriptores: Migración; rendimiento escolar; evaluación del estudiante; calidad de la educación. (Palabras del Tesauro UNESCO).

\begin{abstract}
The objective of the research was to determine the influence that migration has on the academic performance of students in terms of being a Bachelor. Methodologically, it is a non-experimental correlational descriptive type, using data from 1234 students, belonging to the cantons of Cañar, El Tambo and Suscal of Ecuador. By accepting the affirmative hypothesis, it is verified that the motivation to migrate positively influences the academic performance of the students. Migration, when planned, can positively influence the academic performance of high school students, since they perceive themselves to be able to excel, along with generating opportunities to enter university studies in the host country.
\end{abstract}

Descriptors: Migration; academic achievement; student evaluation; educational quality. (Words from the UNESCO Thesaurus).

\section{INTRODUCCIÓN}

Desde sus inicios la humanidad viene experimentado diferentes fenómenos sociales que afectan directa o indirectamente el entorno en el que se presenta dicho acontecimiento, entre ellos está la migración, que a nivel mundial es conocido por todos los beneficios y consecuencias que el mismo conlleva. El continente americano no es la excepción, el mismo tiene un país que recibe emigrantes de todo el mundo como lo es 
Los Estados Unidos de Norteamérica. En consecuencia, es una potencia mundial, de manera que sobresalta la cultura, sociedad y sobre todo la economía de sí mismo, del continente y del mundo en general.

Desde los años noventa en el Ecuador se produjo una crisis económica que azoto todo el territorio; siendo la clase media baja la más afectada, es por ello que en esos años se propagó la migración interna e internacional, a causa de ello hubo un sin número de repercusiones entre las más perceptibles están la pobreza, la ruptura del núcleo familiar, pérdida de valores, educación inconclusa y el escaso interés por la preparación cultural e intelectual. Sin embargo, habría que decir también que existieron beneficios como las remesas monetarias, mejorar la calidad de vida, nuevas experiencias dentro del ámbito profesional e individual, constituyéndose la migración en un fenómeno de dignidad humana (Aldana-Zavala \& Isea, 2019).

Este artículo se enfoca en el rendimiento académico de los estudiantes que se encuentran por culminar sus estudios de bachillerato, teniéndose en la migración como un factor de incidencia asociado a la misma. Sin embargo dentro del sistema educativo ecuatoriano, se dan procesos muy notables enfocados en destrezas con criterio de desempeño, indicadores de logro, criterios de evaluación y evaluación en base estructurada, dicho de otra manera existe muchos cambios subjetivos pero no significativos, en cuanto a que los estudiantes de bachillerato desarrollan destrezas para solucionar problemas de la vida, mientras que la universidad los prepara en competencias para su vida profesional.

Mientras tanto la migración toma fuerza en los jóvenes que mediante este fenómeno social, perciben que pueden mejorar su calidad de vida, a través de una considerable remuneración y es por ello que solo esperan cumplir su mayoría de edad para emigrar. Además, otra causa que conlleva a tomar esta difícil decisión en el Ecuador, es por las evaluaciones ser bachiller, en la cual para poder obtener acceso la educación universitaria, se debe obtener una calificación muy por encima de la media.

Prosiguiendo con lo planteado, (Tacuri-Vélez, 2016) manifiesta que América latina tiene un gran indice migratorio, siendo los mas pobres quienes abandonan su pais mara 
mejorar su estio de vida. La realidad del Ecuador y en especial de la provincia del Cañar que "egún los datos de la Fondos de las Naciones Unidas para la Infancia (UNICEF) en los ultimos 15 años se ha triplicado la migracion tanto masculina como femenina (Tacuri-Vélez, 2016). Es por ello que existe muchos jóvenes estudiantes son representados por familiares, los mismos que en su gran mayoría no cumplen con las responsabilidades enmarcadas dentro de los estatutos y del Código de Convivencia de las instituciones. Debido a esta problemática el rendimiento académico atraviesa un proceso de vulnerabilidad, por cuanto el estudiante presenta múltiples problemáticas tanto social, psicológico y académicamente.

En este sentido, se tendría que asociar la migración con la educación, en todos sus niveles, dado que en el Ecuador existe un alto nivel de desempleo como da a conocer (Feijoó \& Del Pozo, 2020) Encuesta Nacional de Empleo, Desempleo y Subempleo (ENEMDU), que la tasa de desempleo es del $3,8 \%$ y un $10,9 \%$ de empleo no remunerado a nivel nacional. En definitiva año tras año se incrementa dicho índice, mismo que repercute en los adolescentes que no encuentran una motivación para continuar con sus estudios universitarios, por cuanto; la transición a la universidad se encuentra plagada de adversidades, de donde se infiere que existe desfases en los contenidos, currículo generalizado, instituciones educativas con déficit de infraestructura y sin acceso a recursos tecnológicos, evaluación ser bachiller la misma que selecciona estudiantes de acuerdo a una calificación cuantitativa que no refleja el conocimiento, destrezas, habilidades y potencialidades, es por esto que existe una escasa estimulación para con sus estudios de tercer nivel.

Es probable que la educación sea la mejor manera de luchar contra la pobreza, por el contrario, migrar es la forma más rápida de mejorar los ingresos económicos, sin embargo, los riesgos que ello conlleva son numerosos y muy peligroso, a pesar de lo dicho anteriormente el índice migratorio en el país es del 6, 95\% lo cual refleja que existe una continuidad migratoria. La migración es el resultado de diferentes factores la mayoría de ellos agobiantes, ya que; repercute en el individuo que migra, es por ello que se desarrolla una mescla de identidades (Henríquez, Urzúa, \& López, 2020). Es 
necesario recalcar lo que manifiesta (Gandini, 2018) existe un gran índice de migración de personas con niveles educativos superiores, que realizan labores que no están acordes con sus conocimientos académicos, esto es algo muy frustrante en la personalidad del individuo, debido a que se vuelve un ser reprimido y confundido por tan abrupto cambio, al mismo tiempo intenta cubrir su frustración con la remuneración recibida.

Algo semejante ocurre con la perspectiva que presentan los jóvenes, más aún cuando existe una desigualdad de condiciones dentro del contexto familiar, por cuanto no todos tienen las mismas ambiciones, (Herrera \& Pérez- Martínez, 2015), en su artículo ¿Tiempos de crisis, tiempos de retorno? Trayectorias migratorias, laborales y sociales de emigrantes retornados en Ecuador, manifiesta que la persona es vista como una fuente de egreso dentro del contexto familiar al no disponer de ingresos económicos. Igualmente (Lotero-Echeverri \& Pérez- Rodríguez, 2019) dice que la calidad de vida es directamente proporcional a la situación económica en el interior de la misma familia. Habría que decir también que en la actualidad se viene manejando el término rendimiento académico para hacer referencia a los avances en conocimientos que se tiene dentro del aula de clase, sin embargo dicho resultado puede ser muy subjetivo, dado que la diversidad de conocimientos que posee cada estudiante es infinita, como (Valle-Arias, Regueiro-Fernandez, Suárez- Fernández, Núñez- Pérez, \& Rosário, 2017) manifiesta en su artículo Rendimiento académico que los enfoques de trabajo e implicación en los deberes escolares al estudiante se lo tiene que valorar desde su entorno, el tipo de alegrías, problemas, motivación, triunfo, metas incluso en la realización de una actividad sencilla, mismo que ayudara a encontrar estrategias que ayuden a cumplir metas trazadas (Roque-Díaz, 2018). Hay que mencionar, además que cada individuo aprende de manera diferente, además de la motivación que muestra por el continuar con su preparación académica e intelectual, a su vez (Bustos-González. \& Gairín-Sallán., 2017). Menciona que todo nace en el interior del estudiante, del cómo se prepara para llegar a las metas propuestas y así lograr sus objetivos de vida. 
Lo dicho hasta aquí supone que la migración es un fenómeno altamente peligroso para la comunidad en sí, de manera puntual me refiero a los jóvenes que no dudan en migrar, en especial desde sectores rurales, hacia lugares urbanos o metrópolis, lo que produce un alto rango de deserción escolar, de ahí que (Alvarado-López, CorreaQuezada, \& Tituaña-Castillo, 2017) manifiesta que el objeto principal de la migración es la necesidad, en definitiva los términos están ligados de manera objetiva, ya que son conceptos colíndales.

Como describe la UNICEF, Cañar está entre los diez cantones con mayor incidencia de emigración, de preferencia hombres que están entre los 18 y 49 años de edad, según la encuesta cantonal el $60 \%$ de los emigrantes dejaron hijos e hijas, y de estos el $60 \%$ tienen menos de 18 años, siendo los del sector rural con $85 \%$ los que más migran. El fenómeno migratorio es un reto para la educación, los mismos tienen principios de integración, igualdad e interculturalidad (Pérez-Velasquez, 2017). Por esta razón que la educación se vuelve una preocupación en la que se tiene que invertir recursos, sacrificio individual y colectivo además de paciencia para que después de algunos años se tenga un trabajo digno que permita la reinversión social una y otra vez (Fidel-Castro, Albán-Yánez, Quizphe- Baculima, Lara-Olalla, \& Portelles-Cobas, 2017). Haciendo referencia a esta idea America Latina debe formar el capital humano entre una población de jóvenes y proporcionarles oportunidades en el mercado laboral.

Desde esta perspectiva el Gobierno Nacional a través del Instituto Nacional de Evaluación Educativa del Ecuador (INEVAL) en el 2013 implemento la evaluación a los alumnos del Tercer Año de Bachillerato, como requisito para graduarse, esta evaluación es conocida como "Ser Bachiller" dicha evaluación se elabora a través de los estándares establecidos por el Ministerio de Educación y el Instituto Nacional de Evaluación Educativa (INEVAL), dicha evaluación involucra el promedio de la nota obtenida en el dominio social, matemático, lingüístico, científico y abstracto, los resultados que se han obtenido presenta una evidencia en el distrito 03D02 el promedio en el periodo lectivo 2018-2019 fue de 8,06 mismo que hace referencia al criterio de "satisfactorio" (INEVAL, 2017). 
Bajo este contexto, se pretende analizar mediante un estudio de caso, en el conocimiento del rendimiento académico de los alumnos del tercer año de bachillerato pertenecientes a los cantones que tienen mayor índice migratorio dentro de la provincia del Cañar durante el periodo lectivo 2018-2019, avanzando en nuestro razonamiento, se debe agregar lo manifestado por (Álvarez-de-Sotomayor-Posadillo, 2015) la integración educativa se debe analizar desde una dimensión sociocultural, esto es el contacto cultural.

Considerando que la migración es un fenómeno que afecta a todos los individuos de diferentes maneras ya sea en el aspecto social, cultural, académico, afectivo y económico nos vemos en la necesidad de dar a conocer el significado de migración según (McAdam, 2014) "La migración a causa de las crisis debe ser entendida en términos de "puntos de inflexión" que se desencadenan no solo por eventos sino también por procesos estructurales subyacentes" (p.11). Es necesario recalcar que al ser un análisis migratorio, se debe tener en cuenta aspectos como: la diferencia salarial, demanda de trabajo en otros lugares, expectativas económicas y sociales (Oyarzun, 2008). En función de lo abordado, el objetivo del presente trabajo se centró en determinar la influencia que tiene la migración en el rendimiento académico de los estudiantes en cuanto a la evaluación Ser Bachiller.

\section{MÉTODO}

Para el análisis del rendimiento académico en el Distrito de Educación 03D02 Cañar-El Tambo-Suscal se partió de los resultados en las Pruebas Ser bachiller lectivo 20182019, realizada por el Instituto Nacional de Evaluación Educativa (INEVAL, 2019). La prueba se realizó a los estudiantes del Tercer Año de Bachillerato a nivel nacional obteniendo un promedio de 7,70 puntos sobre 10 , es decir, a nivel nacional los estudiantes se ubican en un nivel de logro Elemental.

Las cifras utilizados fueron recolectados de una fuente segura y confiable como lo es él INEVAL, es por ello que la metodología es tipo descriptiva correlacional no 
experimental, a través de los datos de 1234 estudiantes, perteneciente a los cantones de Cañar, El Tambo y Suscal, de los cuales 618 son hombres y 616 mujeres que pertenecen a 20 Instituciones Educativas, de donde resulta que 17 tienen sostenimiento fiscal, 2 fiscomisional y 1 particular, además 11 de ellas se encuentran en el área rural y las 9 restantes en el área urbana.

Siendo transversal, pues la información se la recolectó en Diciembre del 2019, con el propósito de brindar un panorama del logro académico alcanzado por los estudiantes en los campos que fueron evaluados como son: Dominio Científico, Dominio Matemático, Dominio Social y Dominio Lingüístico, el puntaje obtenido sirve para postularse la educación superior, como está estipulado en la ficha técnica del (INEVAL, 2017).

Se debe agregar el tipo de sostenimiento, el área y jurisdicción, por lo que se refiere a los índices migratorios los datos recolectados son: Cañar 9,16\%, El Tambo 11,09\% y Suscal $10 \%$. Una vez recogida la información se realizó un análisis estadístico descriptivo que muestra las frecuencias y los porcentajes de las variables de interés, así como se empleó el Chi-cuadrado de Pearson para establecer la asociación entre las variables, mediante análisis del Software IBM SPSS Stadistics 20 (Argudo-Tello, ErazoÁlvarez, \& Narváez-Zurita, 2019).

\section{RESULTADOS}

A continuación, se presentan los resultados de las variables trabajadas, las que se relacionan también con información demográficas de las diferentes instituciones educativas: 
Revista Arbitrada Interdisciplinaria KOINONIA

Año 2020. Vol V. ํ⒈ Especial Educación

Hecho el depósito de Ley: FA2016000010 ISSN: 2542-3088

FUNDACIÓN KOINONIA (F.K). Santa Ana de Coro. Venezuela.

Mónica Carolina Ojeda-Chimborazo; Darwin Gabriel García-Herrera; Juan Carlos Erazo-Álvarez; Cecilia Ivonne Narváez-Zurita

\section{Tabla 1}

Rendimiento académico

\begin{tabular}{cccccc} 
& & Frecuencia & Porcentaje & Porcentaje válido & Porcentaje acumulado \\
\hline \multirow{4}{*}{ Válidos } & $5,00-5,99$ & 10 & 0,8 & 0,8 & 0,8 \\
& $6,00-6,99$ & 108 & 8,7 & 8,8 & 9,6 \\
& $9,00-10,00$ & 128 & 10,4 & 10,4 & 19,9 \\
& $7,00-7,99$ & 430 & 34,8 & 34,8 & 54,8 \\
& $8,00-8,99$ & 558 & 45,1 & 45,2 & 100,0 \\
\multicolumn{1}{c}{ Perdidos } & Total & 1234 & 99,8 & 100,0 & \\
\multicolumn{2}{c}{ Total } & 2 & 0,2 & & \\
\hline
\end{tabular}

Fuente: Elaboración propia

En lo que hace referencia al rendimiento académico se puede analizar en la tabla 1, de la misma se puede manifestar que alrededor de la mitad los estudiantes tienen un nivel de logro Satisfactorio, es decir mayor al promedio nacional, además su desviación estándar es de 0,824 lo que demuestra que el nivel de logro de los estudiantes es más que elemental, sin dejar a un lado un porcentaje del 9,6\% que está por debajo del logro elemental.

\section{Tabla 2}

Cantón, Sostenimiento y Área

\begin{tabular}{cccccccc} 
& & \multicolumn{2}{c}{ Fiscal } & \multicolumn{2}{c}{$\begin{array}{c}\text { Sostenimiento } \\
\text { Fiscomisional }\end{array}$} & \multicolumn{2}{c}{$\begin{array}{c}\text { Particular } \\
\text { Área }\end{array}$} \\
& & \multicolumn{2}{c}{ Área } & \multicolumn{2}{c}{ Área } & \multicolumn{2}{c}{ Arrean } \\
& & Urbana & Rural & Urbana & Rural & Urbana & Rural \\
& Recuento & Recuento & Recuento & Recuento & Recuento & Recuento \\
\hline \multirow{3}{*}{ Cantón } & Cañar & 512 & 341 & 129 & 0 & 26 & 0 \\
& El Tambo & 170 & 0 & 22 & 0 & 0 & 0 \\
& Suscal & 34 & 0 & 0 & 0 & 0 & 0 \\
\hline
\end{tabular}

Elaboración: Fuente propia 
Se demuestra que en las instituciones fiscomisional y particular se encuentran en el área urbana, mientras que en las instituciones con sostenimiento fiscal un $39,98 \%$ se encuentran en el área rural y en lo que hace referencia al distrito desciende hasta $27,63 \%$, este dato se puede relacionar con la tasa migratoria, la misma que en el sector rural es mayor que en el urbano.

\section{Tabla 3}

Migración y rendimiento académico por Cantón

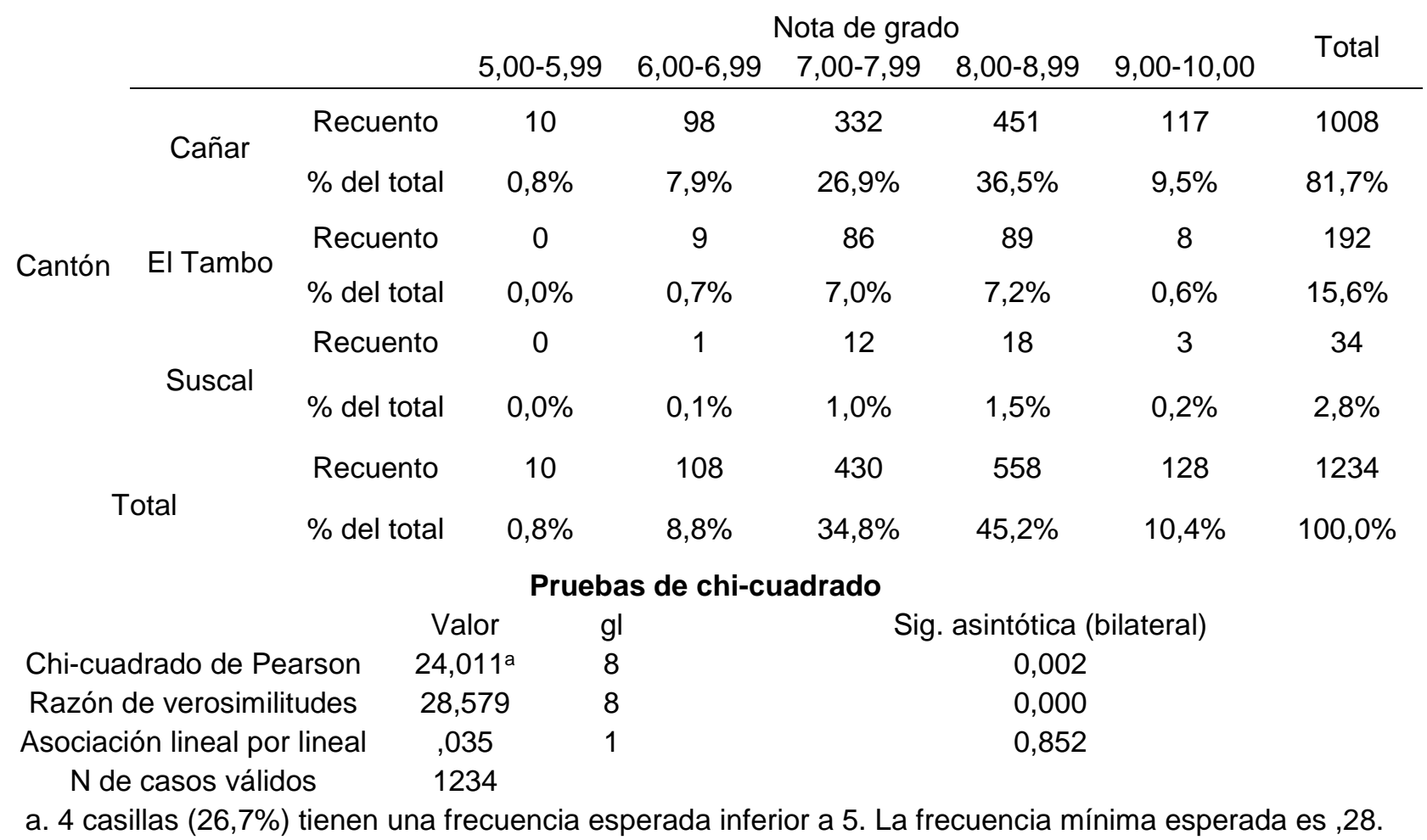

Fuente: Elaboración propia

Con respecto a la migración contamos con los índices migratorios de los tres cantones que pertenecen a este distrito, los mismos son: Cañar 9,16\%, El Tambo 11,09\% y Suscal $10 \%$. Manifestando además que son los índices más altos de la provincia y están entre los 20 primeros cantones con más habitantes que han migrado. 
Se analizó desde Chi-cuadrado de Pearson que manifiesta si el $p$-valor de $a \leq 0,05$ la hipótesis es válida, de lo contrario si $p$-valor de $a \geq 0,05$ la hipótesis es nula, en este sentido el valor de Chi-cuadrado de Pearson es de 0,02 lo cual deja notar la relación que existe entre las variables planteadas.

Prueba de hipótesis

H0: No existe asociación entre migración y rendimiento académico

H1: Existe asociación entre migración y rendimiento académico

Se acepta la hipótesis afirmativa y se rechaza la nula.

\section{DISCUSIÓN}

De acuerdo con los datos obtenidos se determina que el nivel académico demostrado por los estudiantes del Tercer Año de Bachillerato del Distrito de Educación 03D02, en la evaluación Ser Bachiller 2018-2019 son superiores al logro elemental, llegado a este punto se tiene que manifestar que 468 estudiantes de 1234 tiene un nivel de logro insuficiente, siendo $37,82 \%$ del total, además que el promedio de migración de los cantones pertenecientes al distrito es del $10,08 \%$ se puede inferir que la migración afecta indirectamente a la educación, como bien lo dice (Fernández-Guzmán, 2013), al producir transformaciones económicas y sociales, contribuye para que exista una nueva generación migrante, donde la única premisa seria el dinero.

Además el entorno social juega un papel preponderante debido a que el promedio de las nota de grado en el sector urbano es de $8,19 \%$ (893 estudiantes) mientras que en el sector rural desciende a $7,73 \%$ (341estudiantes), superando la media nacional, sin embargo, se tiene que mencionar además la brecha que existe entre las instituciones según su área geográfica, teniendo en cuenta lo planteado por (Solís-Lizama, 2018), la fuerza laboral más solicitada y mejor remunerada de los inmigrantes es aquella procedente sectores lejanos, los mismos que demuestran su necesidad a través del esfuerzo físico, queriendo demostrar ser reciproco con la remuneración.

Al aceptarse la hipótesis afirmativa, se comprueba que la motivación a migrar influye positivamente en el rendimiento académico de los estudiantes, por cuanto perciben que 
podrían tener mayores oportunidades de estudiar y de empleo, con la finalidad de mejorar la calidad de vida, lo cual concuerda con los planteamientos (Fajardo-Bullón, et al., 2017).

\section{PROPUESTA}

El siguiente punto trata de dar una respuesta al problema suscitado, por los resultados adquiridos en esta investigación, se ha podido evidenciar que existe un sin número de problemas que afectan el rendimiento académico de los estudiantes en general dentro y fuera de las instituciones educativas, probablemente sea difícil dar solución a todos los problemas, pero se ha creído conveniente trabajar con cuatro pilares fundamentales, los mismos ayudaran dentro del proceso enseñanza aprendizaje ellos son: las necesidades que tienen los estudiantes, resultados a ser alcanzados por los miembros de la Unidad Educativa, el proceso de enseñanza debe permanecer en constante monitoreo e identificar las fortalezas de cada uno de los estudiantes que posee el plantel, de esta manera se ayudara en el progreso del rendimiento académico. Además, se ha trabajado en un esquema él mismo se muestra a continuación:

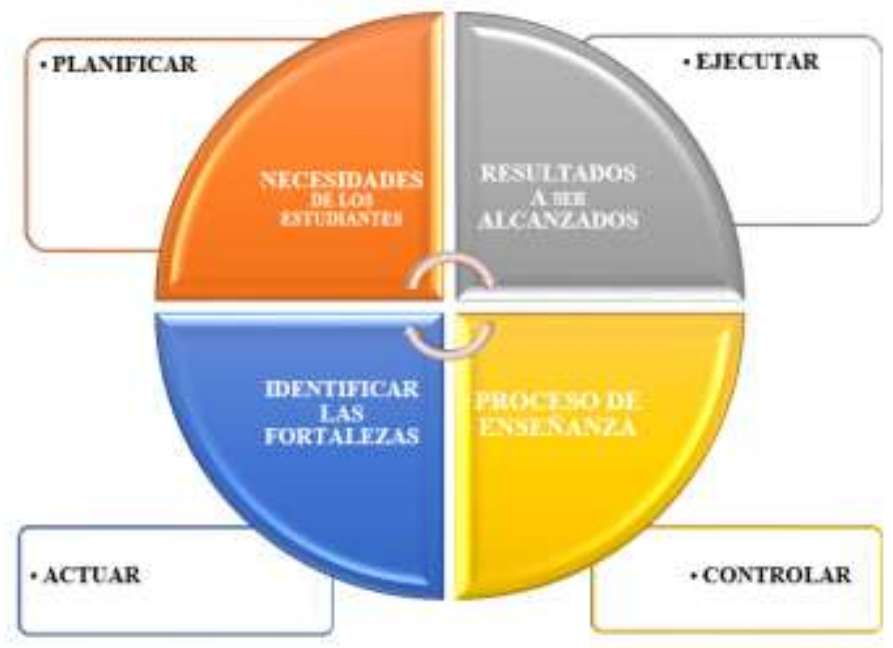

Figura 1. Pilares para mitigar el rendimiento académico. Fuente: Elaboración propia. 
A continuación, se detalla cada uno de los pilares.

Planificar: Se parte de la autenticidad de cada estudiante, su entorno, su contexto, su mundo, su forma de pensar, las aspiraciones que tiene en la vida y sobre todo las necesidades que posee, ya sea sociales, económicas o culturales, por todo esto la importancia de la visión, misión, el código de convivencia y las políticas institucionales que se implementa dentro de cada Unidad Educativa. Razón por la cual se plantea una Ficha de Vida Estudiantil "La Cuenta Todo" con el objetivo de conocer al ser humano que está dentro del llamado estudiante, por lo cual se profundiza aspectos que tengan relevancia dentro del contexto familiar y escolar. Por consiguiente, lo que va al inicio es el Encabezado, en el mismo se detalla el nombre de la institución educativa, el periodo lectivo escolar, código de la institución.

Luego los Datos de Identificación, del estudiante, padre, madre y representante legal, en esta parte deberán escribir los apellidos y nombres del estudiante, su lugar y fecha de nacimiento, número de cédula (si es que posee), domicilio, institución educativa de dónde procede, curso en el que actualmente está estudiando y el paralelo, además de datos médicos como si tiene alguna discapacidad, tipo de sangre, el uso de lentes, alergias. Lo que sigue son los Datos Familiares, en esta parte se llenan los datos del padre, de la madre y de ser el caso el representante legal, las preguntas son número de cédula, teléfono, profesión u ocupación y lugar de trabajo.

La segunda parte empieza con el Núcleo Familiar, aquí se debe especificar todas las personas con quien vive el estudiante, a lo mejor alguien ha migrado o retornado. Posteriormente se continúa con los datos socioeconómicos, esta parte hace referencia a las viviendas, vehículos, ingresos y egresos familiares, finalmente la Parte Afectiva, no se trata tan solo del contexto escolar, dado que el estudiante la mayor parte del tiempo permanece en la Institución Educativa se tiene que realizar preguntas que tiene relación con los docentes, si le gusta el estudio, tiene amigos, le maltratan en el hogar, en la institución, quienes son los que maltratan, como lo hacen y cualquier actividad que le afecte emocionalmente. Ver anexo. 
Ejecutar: inicia el día en el que es estudiante ingresa al establecimiento educativo, en todos los subniveles, la o el tutor de curso tendrá que llenar la ficha "La Cuenta Todo" al inicio del periodo lectivo, y se la tendría que volver a llenar al iniciar el segundo quimestre, es necesario recalcar que el objetivo de dicha actividad es mejor el proceso enseñanza-aprendizaje desde una perspectiva social y un clima familiar, teniendo en cuenta que los datos registrados servirán para conocer el entorno del estudiante, como resultado de los datos adquiridos durante los dos procesos.

Controlar: para tener un conocimiento real de los datos, es necesario contrastarlos los del inicio del año lectivo con los de la mitad del año lectivo y finalmente con los del nuevo año lectivo, para ello es necesario un trabajo en equipo entre el docente saliente y el docente entrante, teniendo en cuenta que durante diez meses trabajo con todos los miembros del salón, además asimilo todos los problemas que se desarrolló durante el lectivo, de manera puntual me refiero al procesos de transmisión de conocimientos, o a su vez detectar el problema para que no exista dicha trasmisión.

Actuar: es la fase de seguimiento que se da en el transcurso del periodo lectivo, en consonancia con la información recolectada y los procesos desarrollados durante las jornadas educativas. Todas estas observaciones se relacionan también con lo que sucede dentro del estudiante, sus aspiraciones, motivaciones y ganas de superación. El saber seleccionar las fortalezas que posee y convertirlas en oportunidades, con el fin de mejorar en el aspecto académico, educativo, social y cultural en definitiva vivir el Sumak Kawsay.

\section{CONCLUSIÓN}

La migración cuando es planificada, puede influir positivamente en el rendimiento académico de los estudiantes de bachillerato, por cuanto se perciben con posibilidades de superarse, aunado a generar oportunidades de ingresar a estudios universitarios en el país receptor, así como la posibilidad de contar con competencias académicas en pro de lograr empleos en mejora de la calidad de vida, pudiendo así poder diseñar proyecto de vida a partir del crecimiento educativo. 


\section{FINANCIAMIENTO}

No monetario.

\section{AGRADECIMIENTO}

Al personal Directivo y Docente de las Escuelas cantones de Cañar, El Tambo y Suscal.

\section{REFERENCIAS CONSULTADAS}

Aldana-Zavala, J., \& Isea, J. (2019). Migración. Un fenómeno del siglo XXI. IUSTITIA SOCIALIS, 4(6), 52-70. http://dx.doi.org/10.35381/racji.v4i6.288

Alvarado-López, J., Correa-Quezada, R., \& Tituaña-Castillo, M. (2017). Migración interna y urbanización sin eficiencia en países en desarrollo: evidencia para Ecuador [Internal migration and urbanization without efficiency in developing countries: evidence for Ecuador]. Papeles de población(94), 99-123. doi:DOI: http://dx.doi.org/10.22185/24487147.2017.94.033

Álvarez-de-Sotomayor-Posadillo, A. (2015). El rendimiento académico de los alumnos inmigrantes en españa: un estudio de caso [The academic performance of immigrant students in Spain: a case study]. Universidad de Granada. Obtenido de https://n9.cl/3pqb1

Argudo-Tello, K. J., Erazo-Álvarez, J. C., \& Narváez-Zurita, C. I. (2019). Evaluación de Control Interno en Riesgos Estratégicos para la Dirección de Planificación de la Universidad de Cuenca. [Evaluation of Internal Control in Strategic Risks for the Planning Direction of the University of Cuenca]. Revista Arbitrada Interdisciplinaria Koinonía, 4(1), 67-96. http://dx.doi.org/10.35381/r.k.v4i1.372

Bustos-González., R., \& Gairín-Sallán., J. (2017). Expectativas académicas de estudiantes y padres migrantes: el caso de Arica en la frontera de Chile y Perú [Academic expectations of migrant students and parents: the case of Arica on the border of Chile and Peru.]. Revista Dilemas Contemporáneos: Educación, Política y Valores.(3), 1-28. Recuperado de https://goo.gl/Q4Kt7d

Fajardo-Bullón, F, Maestre-Campos, M, Felipe-Castaño, E, León-del-Barco, B, \& Polodel-Río, M. (2017). Análisis del rendimiento académico de los alumnos de educación secundaria obligatoria según las variables familiares. Educación XX1, 20 (1), 209-232. Recuperado de https://cutt.ly/SiYBhds 
Feijoó, E., \& Del Pozo, D. (2020). Encuesta Nacional de Empleo, Desempleo y Subempleo (ENEMDU) [National Survey of Employment, Unemployment and Underemployment (ENEMDU)]. Recuperado de https://cutt.ly/oiYZPFI

Fernández-Guzmán, E. (2013). Migración internacional en un pueblo michoacano. Retorno e inversión migrante (1982-2008): El caso Huandacareo [International migration in a town in Michoacán. Return and migrant investment (1982-2008): The Huandacareo case]. Agricultura, sociedad y desarrollo, 10(3). Recuperado de https://n9.cl/45zj

Fidel-Castro, B., Albán- Yánez, H., Quizphe- Baculima, V., Lara- Olalla, M., \& PortellesCobas, D. E. (2017). El Proceso Educativo Estatal y las Pruebas Ser Bachiller [The State Educational Process and the Tests to Be a Bachelor]. Investigación Talento, 70 - 74. Recuperado de https://n9.cl/i1iq

Gandini, L. (2018). Migración de alta educación, desarrollo y derechos humanos ¿la incógnita de la ecuación? [Migration of high education, development and human rights: the unknown of the equation]. Iztapalapa. Revista de ciencias socialesy humanidades, 39(84),

75-103. http://dx.doi.org/10.28928/revistaiztapalapa/842018/atc3/gandinil.

Henríquez, D., Urzúa, A., \& López, W. (06 de 04 de 2020). Fusión de identidad en migrantes: análisis psicométrico de la escala verbal [Identity fusion in migrants: psychometric analysis of the verbal scale]. Suma Psicológica, 26(2), 86-93. http://dx.doi.org/10.14349/sumapsi.2019.v26.n2.4

Herrera, G., \& Pérez- Martínez, L. (2015). ¿Tiempos de crisis, tiempos de retorno? Trayectorias migratorias, laborales y sociales de migrantes retornados en Ecuador [Times of crisis, times of return? Migratory, labor and social trajectories of returned migrants in Ecuador]. Estudios Políticos(47), 221-241. Recuperado de https://cutt.ly/6iYHnAi

INEVAL. (2017). Ficha técnica y conceptual [Technical and conceptual data sheet]. Recuperado de https://n9.cl/y316

INEVAL. (2019). Informe de resultados Nacional Ser Bachiller año lectivo 2018-2019 [Report of results National Be Bachelor school year 2018-2019]. Quito-Ecuador. Recuperado de https://n9.cl/y316 
Lotero-Echeverri, G., \& Pérez- Rodríguez, M. A. (2019). Migraciones en la sociedad contemporánea: Correlación entre migración y desarrollo [Migrations in contemporary society: Correlation between migration and development]. Retos. Revista de Ciencias de la Administración y Economía, 9(17), 145-159. https://doi.org/10.17163/ret.n17.2019.09

McAdam, J. (2014). El concepto de migración a causa de las crisis [The concept of migration due to crises]. Revista Migraciones Forzadas, 45. Recuperado de http://hdl.handle.net/10045/36440

Oyarzun, J. (2008). Causas y efectos económicos de la inmigración. Un análisis teóricoempírico [Causes and economic effects of immigration. A theoretical-empirical analysis]. Papeles del este, 17, 2-40. Recuperado de https://n9.cl/s89n

Pérez-Velasquez, A. (2017). Incidencia de la migracion en el rendimiento academico en los niños de sexto año de educacion basica [Incidence of migration on academic performance in children of the sixth year of basic education]. Universidad Politécnica Salesiana, Cuenca. Recuperado de https://n9.cl/jukf

Roque-Díaz, R. (2018). Desarrollo personal y manejo asertivo de emociones en estudiantes. [Personal development and assertive management of emotions in $\begin{array}{llll}\text { students]. } & \text { EPISTEME }\end{array}$ http://dx.doi.org/10.35381/e.k.v1i2.511

Solís-Lizama, M. (2018). Labor Reintegration of Return Migrants in Two Rural Communities of Yucatán, Mexico [Labor Reintegration of Return Migrants in Two Rural Communities of Yucatan, Mexico]. Migraciones internacionales, 9(35), Migraciones internacionales. doi:10.17428/rmi.v9i35.416

Tacuri-Vélez, R. N. (2016). Alteración de la conducta en niños de 5-9 años de padres migrantes y no migrantes en la provincia del Cañar comunidad La Capilla. AbrilSeptiembre 2015 [Alteration of behavior in children of 5-9 years of migrant and non-migrant parents in the province]. 1-39. Recuperado de https://n9.cl/eag4

Valle-Arias, A., Regueiro-Fernandez, B., Suárez- Fernández, N., Núñez- Pérez, J., \& Rosário, P. (2017). Rendimiento académico, enfoques de trabajo e implicación en los deberes escolares [Academic performance, work approaches and involvement in schoolwork]. Magis. Revista Internacional de Investigación en Educación, 10(20), 123-142. Recuperado de https://n9.cl/1puq 
Revista Arbitrada Interdisciplinaria KOINONIA

Año 2020. Vol V. ํ⒈ Especial Educación

Hecho el depósito de Ley: FA2016000010 ISSN: 2542-3088

FUNDACIÓN KOINONIA (F.K). Santa Ana de Coro. Venezuela.

Mónica Carolina Ojeda-Chimborazo; Darwin Gabriel García-Herrera; Juan Carlos Erazo-Álvarez; Cecilia Ivonne Narváez-Zurita

(C)2020 por los autores. Este artículo es de acceso abierto y distribuido según los términos y condiciones de la licencia Creative Commons Atribución-NoComercial-CompartirIgual 4.0 Internacional (CC BY-NC-SA 4.0)

(https://creativecommons.org/licenses/by-nc-sa/4.0/).

\section{ANEXO}

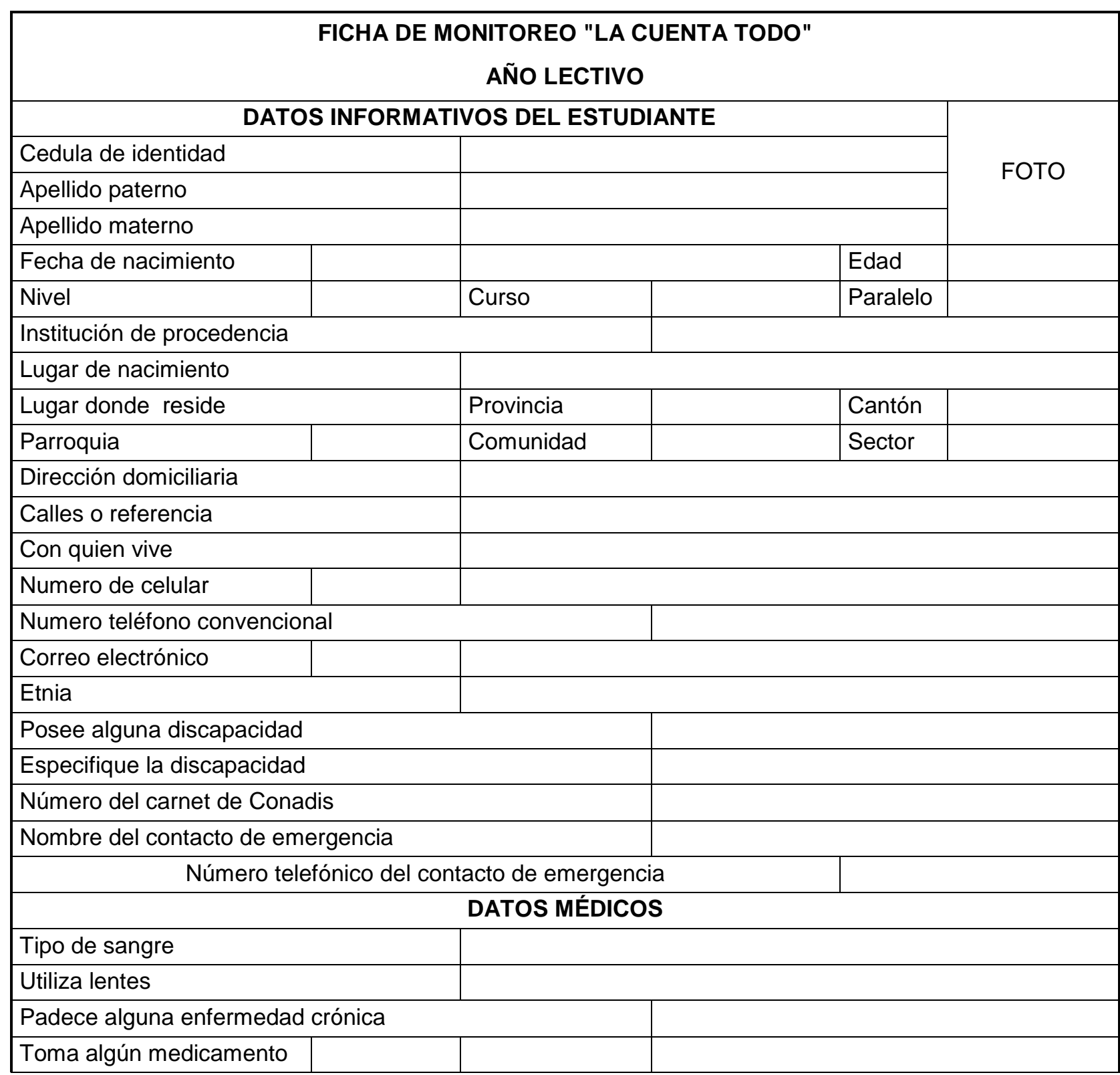


Revista Arbitrada Interdisciplinaria KOINONIA

Año 2020. Vol V. ํ⒈ Especial Educación

Hecho el depósito de Ley: FA2016000010 ISSN: 2542-3088

FUNDACIÓN KOINONIA (F.K). Santa Ana de Coro. Venezuela.

Mónica Carolina Ojeda-Chimborazo; Darwin Gabriel García-Herrera; Juan Carlos Erazo-Álvarez; Cecilia Ivonne Narváez-Zurita

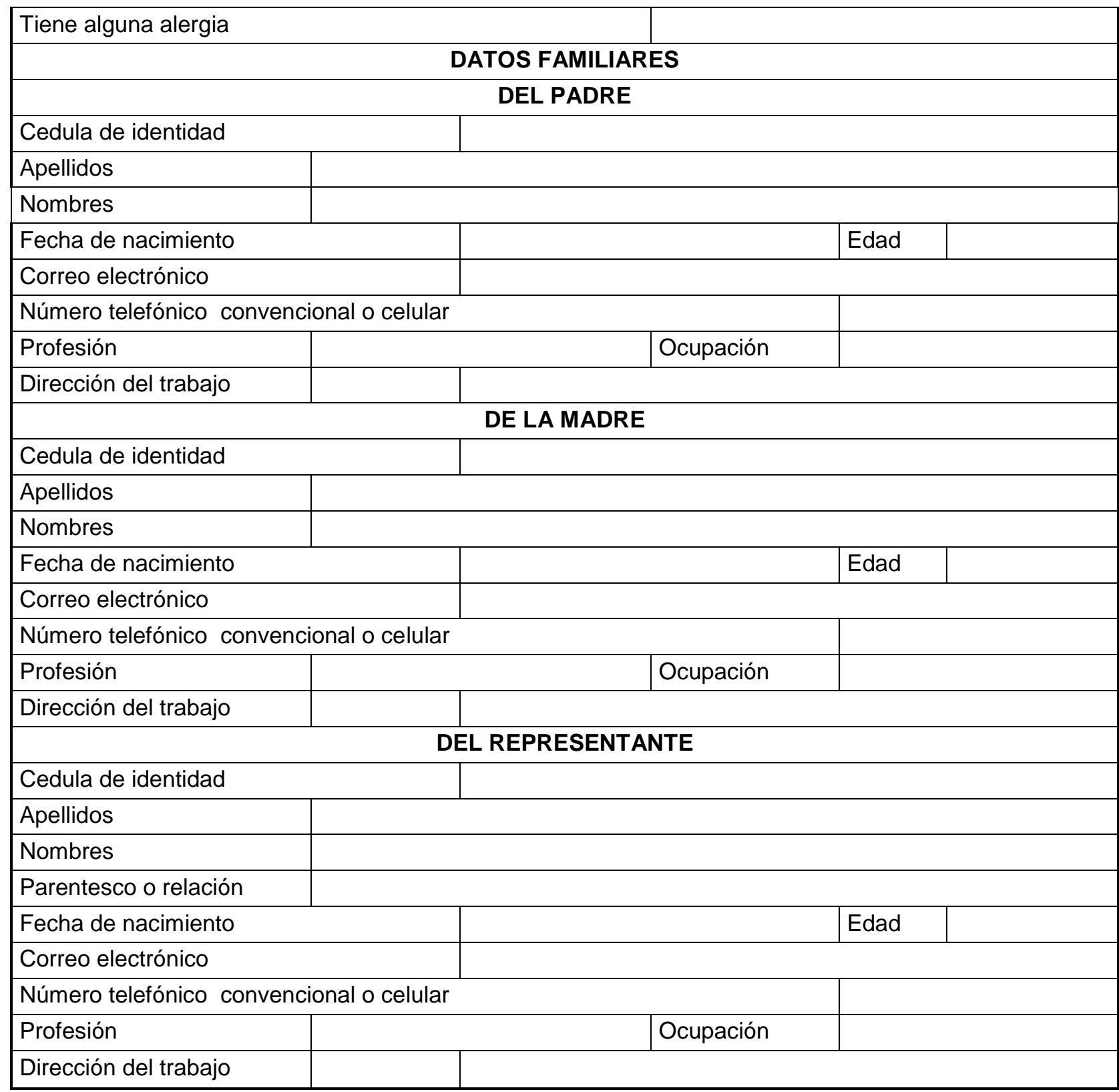

\begin{tabular}{|l|l|l|l|}
\hline \multicolumn{4}{|l|}{ NÚCLEO FAMILIAR } \\
\hline Numero de hermanos & \multicolumn{1}{|l|}{} \\
\hline Lugar que ocupa entre los hermanos & Edad & $\begin{array}{c}\text { NIVEL DE } \\
\text { ESTUDIO }\end{array}$ & CURSO \\
\hline Nombre de los hermanos & & & \\
\hline & & & \\
\hline
\end{tabular}


Revista Arbitrada Interdisciplinaria KOINONIA

Año 2020. Vol V. ํ⒈ Especial Educación

Hecho el depósito de Ley: FA2016000010 ISSN: 2542-3088

FUNDACIÓN KOINONIA (F.K). Santa Ana de Coro. Venezuela.

Mónica Carolina Ojeda-Chimborazo; Darwin Gabriel García-Herrera; Juan Carlos Erazo-Álvarez; Cecilia Ivonne Narváez-Zurita

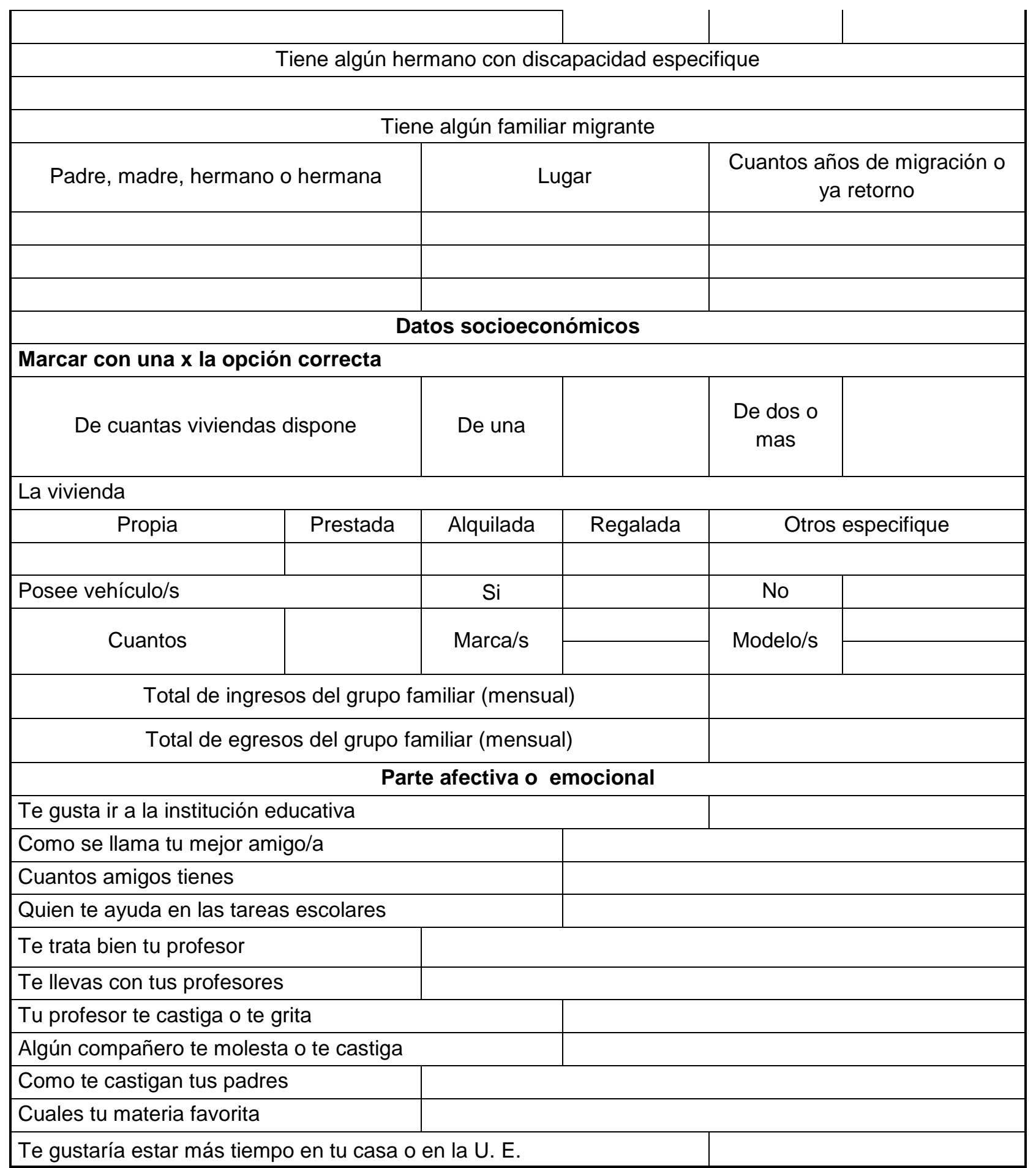

